\title{
Ô nhiễm rác thải nhựa: vấn đề lớn của nhân loại trong thế kỷ 21
}

\author{
Phạm Hà Trang, Hoàng Trường Giang, Nguyễn Cẩm Dương
}
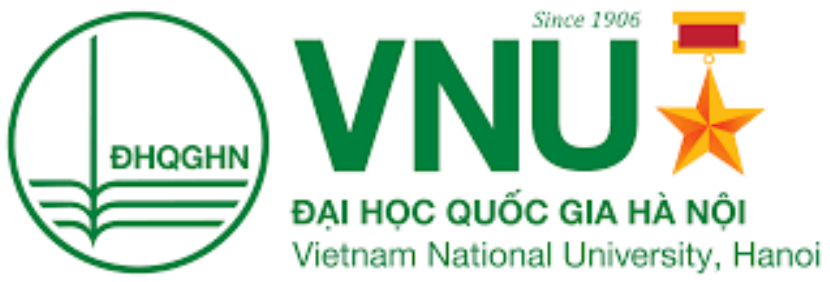

Đại học Quốc gia Hà Nội, Việt Nam

Hà Nội, ngày 11 tháng 01 năm 2022

\section{Preprint DOI: 10.31219/osf.io/693df}

Loài người đang đối diện với rất nhiều thách thức to lớn trong thế kỷ 21. Từ biến đổi khí hậu (Q.-H. Vuong, 2021), đến ô nhiễm nguồn nước (Hanh, 2022), năng lượng , ô nhiễm không khí (Van Khuc et al., 2020; Q. Vuong et al., 2021), mất rừng và suy thoái rừng (Khuc et al., 2018), đói nghèo, bệnh dịch . Trong bài này, chúng tôi trình bày một vấn đề lớn khác đó là ô nhiễm rác thải nhựa (Peng et al., 2021).

Như chúng ta đã biết đồ dùng, vật dụng nhựa được biết đến với rất nhiều lợi ích nên dẫn đến tình trạng sản xuất với số lượng lớn làm chúng ta ngày nay phải đối mặt với một vấn đề nghiêm trọng - rác thải nhựa đặc biệt là sản phẩm nhựa dùng một lần. Rác thải nhựa có mặt ở khắp nơi và được báo cáo từ Bắc Cực đến Nam Cực, từ bề mặt đến trầm tích (Barnes et al., 2009). Tuy nhiên, bao bì nhựa cho thực phẩm, đồ uống và các mặt hàng thuốc lá thường chỉ được sử dụng một lần, điều này đã góp phần vào $61 \%$ rác thải bãi biển toàn cầu(Brooks et al., 2018). Bao bì nhựa và các mặt hàng sử dụng một lần xâm nhập vào dòng chất

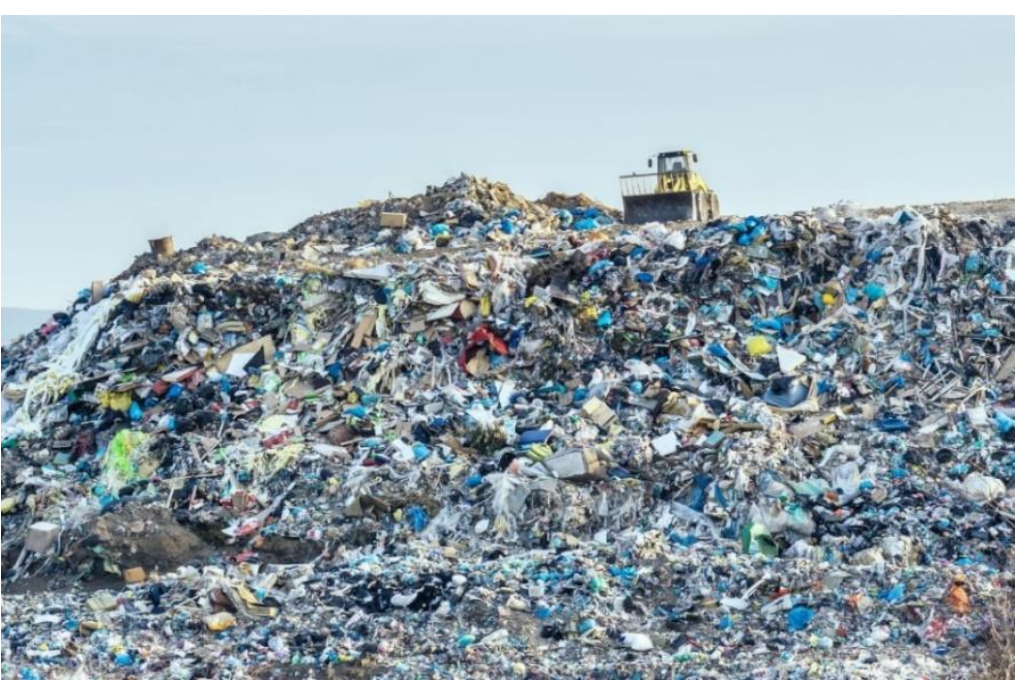

Hình 1: Thực tiễn khi Châu Âu đura ra các muc tiêu tái chế rác thải đầy tham vọng.

Anh: europarl.europa.eu 
thải ngay sau khi sử dụng, góp phần tích lũy hơn 6,3 tỷ tấn chất thải nhựa được tạo ra trên toàn thế giới, chỉ có $9 \%$ chất thải nhựa đã được tái chế trên toàn cầu, với phần lớn chất thải nhựa toàn cầu được chôn lấp hoặc cuối cùng gây ô nhiễm môi trường (80\%), dẫn đến ước tính 4 triệu đến 12 triệu tấn nhựa thải vào đại dương hàng năm.(Jambeck et al., 2015).

Kể từ năm 2019 đến nay, toàn nhân loại đã, đang và sẽ phải đấu tranh với đại dịch toàn cầu COVID-19. Tổ chức Y tế Thế giới đã yêu cầu tăng 40\% sản xuất PPE dùng một lần(WHO, 2020). Nếu dân số toàn cầu tuân thủ tiêu chuẩn một khẩu trang dùng một lần mỗi ngày sau khi lệnh phong tỏa kết thúc, đại dịch có thể dẫn đến tiêu thụ toàn cầu hàng tháng và lãng phí 129 tỷ khẩu trang và 65 tỷ găng tay. Các bệnh viện ở Vũ Hán, trung tâm của dịch COVID-19, đã sản xuất hơn 240 tấn chất thải $\mathrm{y}$ tế sử dụng một lần (như khẩu trang dùng một lần, găng tay và áo choàng) mỗi ngày vào thời kỳ đỉnh điểm của đại dịch, gấp 6 lần so với mức trung bình hàng ngày trước khi đại dịch xảy ra. Nếu sự gia tăng được quan sát thấy ở Vũ Hán đúng ở những nơi khác, Hoa Kỳ có thể tạo ra cả năm chất thải y tế trong 2 tháng(Yudell et al., 2020). Ngoài ra, COVID-19 khiến việc mua hàng trực tuyến trở nên phổ hiến hơn bao giờ hết Nghiên cứu cũng báo cáo sự gia tăng mua sắm trực tuyến từ 1257\% ở các quốc gia như Việt Nam, Ấn Độ, Trung Quốc, Ý và Đức trong cùng thời gian. Một công ty nghiên cứu thị trường, Rakuten Intelligence đã báo cáo mức tăng trưởng cao hơn $50 \%$ so với mức tăng trưởng $20 \%$ hàng năm trong mua sắm trực tuyến ở Mỹ từ tháng 3 đến giữa tháng 4(Rattner, 2020).

Có rất nhiều nguyên nhân dẫn đến ô nhiễm rác thải nhựa. Nguyên nhân căn bản và nghiêm trọng nhất tạo ra rác thải nhựa nằm ở văn hóa môi trường hạn chế của con người $(\mathrm{Q}$. H. Vuong, 2021). Đó là ý thức của con người còn chưa tốt, điều đó được thể hiện qua những hành động rất phổ biến hiện nay: Lạm dụng sử dụng túi nylon, đồ nhựa dùng 1 lần, đặc biệt là trong kinh doanh bởi giá thành rẻ, tiện dụng mà không quan tâm đến đặc tính khó phân hủy của nhựa. Vứt rác bừa bãi, không đúng nơi qui định, không phân loại rác thải: Việc xả rác

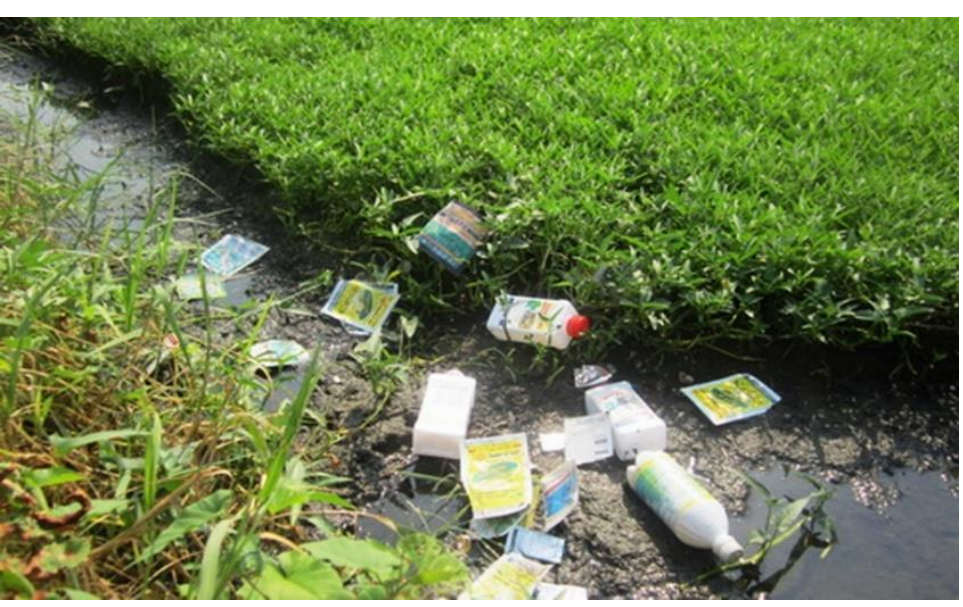

Hình 2: Chất thải nông nghiệp gây ô nhiếm môi truờng nước Anh: quochoi.vn xuống cống rãnh còn gây tắc nghẽn đường ống, làm ngập lụt đường phố. Người dân chưa thật sự quan tâm đến việc phân loại rác thải gây ra nhiều khó khăn.

Tiếp theo, nguyên nhân từ hoạt động nông nghiệp: rác thải đến từ hoạt động nông mang nhiều hóa chất độc hại, gây ô nhiễm nguồn nước, nhiều người nông dân còn “ tiện tay” vứt xuồng ao, sông, mương,...

Nguyên nhân từ các hoạt động y tế: Rác thải từ hoạt động y tế đã qua sử dụng nếu không được xử lý đúng. Đặc biệt, trong dịch bệnh covid vừa qua, lượng rác thải y tế là một áp lực lớn đối với Việt Nam nói riêng và thế giới nói chung, bởi lượng lớn rác thải như áo bảo hộ, khẩu trang, các thiết bị y tế dung 1 lần cho người bệnh,... Nếu không xử lí cẩn thận, rác thải không những ảnh hưởng đến 
môi trường, mà còn có thể là nguy cơ lây lan dịch bênh, hoặc khiến bệnh dịch bùng phát tại một số địa phương.

Nguyên nhân từ các hoạt động du lịch: lượng du khách càng lớn, thì lượng rác thải càng nhiều, gây nên áp lực lớn cho việc thu gom, xử lí rác thải. Đã có nhiều quốc gia buộc phải đóng cửa một số địa điểm du lịch bị ảnh hưởng nghiêm trọng vì rác thải của khách du lịch như Thái Lan, Phillipines,...

Nguyên nhân từ qui trình xử lí rác thải còn nhiều lỗ hổng: Nhiều nước đang phát triển trong đó có Việt Nam, có qui trình xử lí rác thải còn lạc hậu, hiệu suất kém, chưa

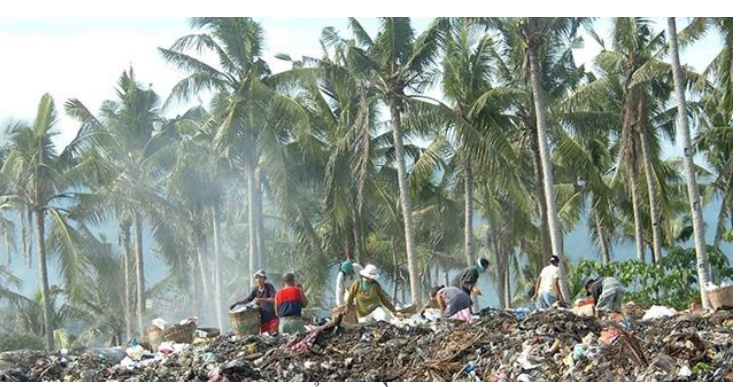

Hình 3: Bãi rác không lồ tại hòn đảo Boracay, Philippines. Anh: AFP xử lí, phân loại, tái sử dụng một cách triệt để.

Nguyên nhân từ sự bàng quan đến từ chính quyền địa phương: Nhiều địa phương chưa thưc hiện đúng các qui định, nghị định, luật lệ ban hành về việc xử lí rác thải, chưa chấp hành nghiêm túc những mức xử phạt hành chính đối với các hành động xả rác bừa bãi không đúng nơi qui định, chưa sát sao trong việc quản lí hệ thống xử lí rác thải.

Nguyên nhân gián tiếp đến từ ngành giáo dục: Giáo dục có vai trò đặc biệt đến chuyển đổi văn hóa (kiến thức, thái độ..) về môi trường, tự nhiên cho thanh niên (Q. Vuong, 2020). Tuy nhiên giáo dục quên đi việc bồi đắp cho học sinh những kĩ năng và ý thức cần thiết nói chung và việc bảo vệ môi trường nói chung. Việc phân loại rác thải, hay vứt rác đúng nơi qui định chưa được sát sao nghiêm khắc, có chăng chỉ là vài buổi thuyết giảng nhàm chán, không được đầu tư để thu hút học sinh và giúp các em hiểu được tầm quan trọng của việc giảm thiểu rác thải.

Rác thải nhựa gây ra rất nhiều hậu quả lớn, Chúng bị thải ra môi trường hoặc bị chôn lấp sẽ bị phân rã thành những mảnh nhựa với kích cỡ khác nhau như: micro, nano, pico... lẫn vào đất, môi trường, không khí khiến cho các loài sinh vật biển, con người ăn phải bị đe dọa đến sức khỏe. Rác thải nhựa xử lý tốt sinh ra các loại khí độc: khí dioxin, furan....sẽ ảnh hưởng rất lớn đến tuyến nội tiết, giảm khả năng miễn dịch, thậm chí gây ung thư. Trong túi nilon có thể lẫn lưu huỳnh, dầu hỏa nguyên chất, đốt cháy gặp hơi nước tạo thành axit sunfuric gây mưa axit, nguy hiểm cho sức khỏe con người. Nhiều sản phẩm nhựa

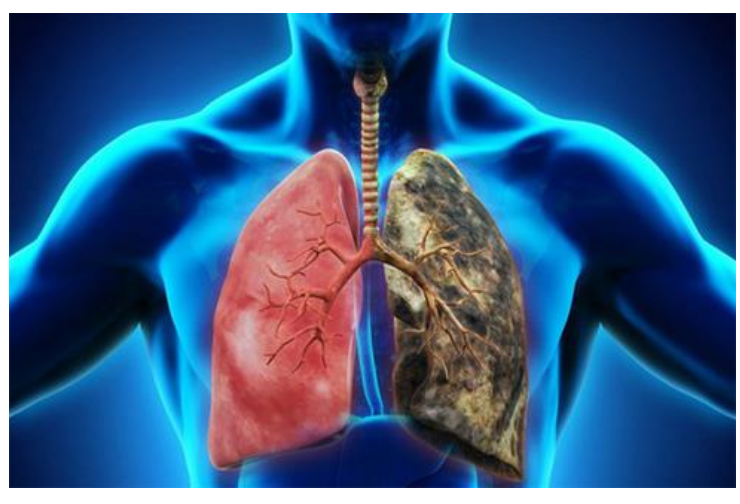

Hình 4: Phổi bị có nguy co bị ung thu Anh: www.vinmec.com kém chất lượng được sản xuất số lượng lớn, trong quá trình sử dụng sẽ sản sinh ra BPA - đây là chất độc hại và gây ra nhiều bệnh lý nguy hiểm ở người như vô sinh, tiểu đường, ung thư...(Tập, 2020b) Đối với môi trường: Sự tồn tại của rác thải nhựa trong môi trường sẽ gây ảnh hưởng tới đất và nước, bởi túi nilon lẫn vào đất sẽ làm thay đổi tính chất vật lý của đất gây xói mòn, làm cho đất không giữ được nước, dinh dưỡng, ngăn cản ôxy đi qua đất, ảnh hưởng đến sinh trưởng của cây trồng. Đối với các loài sinh vật: Rác thải nhựa khi đổ ra biển sẽ gây phá hủy hoặc suy giảm đa dạng sinh học, làm chết các sinh vật biển nếu chúng không may bị mắc vào hoặc ăn phải điều đó làm giảm khả năng hấp thu thức ăn của sinh vật. Khi sinh vật biển ăn phải chất phụ gia trong nhựa và tác động tiêu cực đến 
hệ thống nội tiết và điều hòa hormone trong cơ thể chúng. Các sinh vật biển này có thể chết do nhựa hoặc bị thiếu thức ăn do nhựa đã giết chết các sinh vật trong chuỗi thức ăn của chúng. Đối với những sinh vật biển khi vướng vào lưới đánh cá bị bỏ đi hoặc các loại rác thải nhựa khác, chúng sẽ không thoát ra được nên sẽ yếu dần và chết(Tập, 2020a). Ảnh hưởng đến hoạt động du lịch của con người: khi môi trường bị ô nhiễm thì du khách cũng có ấn tượng không tốt về các điểm du lịch, gây ảnh hưởng đến thu nhập du lịch của địa phương, đất nước, làm khu du lịch không thể phục hồi lại được và trở thành điểm du lịch "chết”'(Tập, 2020a). Ảnh hưởng đến hoạt động khai thác thủy sản của con người: Số lượng sinh vật chết vì rác thải nhựa nhiều thì sản lượng khai thác thủy hải sản giảm. Hơn nữa, rác thải nhựa chặn cửa hút nước hoặc vướng vào lưới đánh cá, cuốn vào chân vịt,... có thể gây hỏng hóc thiết bị.

Mặt khác, đại dịch COVID-19 kéo dài gây ra những tác động sâu sắc về mọi mặt của đời sống xã hội, trong đó có rác thải nhựa (La, 2020). COVID-19 làm gia tăng rác thải nhựa (Peng et al., 2021). Trước tình hình ô nhiễm rác thải nhựa ngày càng trầm trọng, Thủ tướng Chính phủ Việt Nam đã chỉ đạo các văn phòng, cơ quan ngừng sử dụng sản phẩm nhựa dùng một lần vào năm 2020 . Trong quá trình xây dựng dự thảo Chỉ thị hạn chế sử dụng sản phẩm nhựa tại các cơ quan, Bộ đã đánh giá tình trạng lạm dụng sản phẩm nhựa. Thủ tướng Chính phủ đã chỉ đạo các Bộ trưởng, Thủ trưởng cơ quan ngang Bộ, cơ quan thuộc Chính phủ, UBND các tỉnh, thành phố ban hành chỉ thị hoặc kế hoạch xử lí chất thải nhựa từ tháng 10 . Theo kế hoạch, cả nước đặt mục tiêu đến năm 2025 không sử dụng sản phẩm nhựa dùng một lần(Thu Thủy, 2020). Chính phủ Canada đã thực hiện các bước sau để giảm thiểu ô nhiễm nhựa trên phương diện hợp tác: Cấm đồ nhựa sử dụng một lần có hại ngay từ năm 2021 theo Đạo luật Bảo vệ Môi trường Canada, đảm bảo rằng công ty sản xuất sản phẩm nhựa hoặc bán các mặt hàng có bao bì nhựa chịu trách nhiệm quản lý việc thu gom và tái chế chất thải nhựa của họ, làm việc với ngành công nghiệp để ngăn chặn và thu hồi các ngư cụ ma, đầu tư vào các công nghệ mới của Canada, huy động hỗ trợ quốc tế để giải quyết ô nhiễm nhựa, giảm chất thải nhựa từ các hoạt động liên bang(Mont-Saint-Hilaire, 2019)

Tháng 6/2019, Nhật Bản công bố kế hoạch giảm RTN. Chính phủ Nhật Bản đặt mục tiêu sử dụng hiệu quả $100 \%$ các chai nhựa thông qua việc đặt các thùng rác tái chế chuyên dụng cạnh các máy bán hàng tự động. Bên cạnh đó, các cơ quan chức năng Nhật Bản tăng cường tuần tra nhằm ngăn chặn việc đổ rác trái phép. Theo đó, Chính phủ sẽ lắp đặt các thùng chuyên thu gom RTN trên toàn quốc; thúc đẩy các hoạt động vớt rác trên sông, biển; phát triển vật liệu đóng gói phân hủy sinh học(Nguyễn Việt Cường, 2020)...

Để giải quyết những mối đe dọa toàn cầu từ RTN và túi ni lông, năm 2018, Liên hợp quốc đã phát động chủ đề "Giải quyết ô nhiễm nhựa và ni lông" nhằm tuyên truyền, vận động, kêu gọi mọi người cùng thay đổi thói quen sử dụng sản phẩm nhựa dùng một lần, giảm thiểu ô nhiễm môi trường và bảo vệ sức khỏe con người.

Tuy nhiên theo quan điểm của chúng tôi, dưới góc nhìn lý thuyết quản lý 3D (Khuc, 2022; Vuong, Q. H., 2022) và nguyên lý bán dẫn (Q. H. Vuong, 2021), tư tưởng hướng thượng (Khuc, 2021; Nguyen \& Vuong, 2021; Q.-H. Vuong, 2021; Q. H. Vuong et al., 2021), muốn giải quyết vấn đề ô nhiễm môi trường nói chung và rác thải nói riêng cần tập trung vào hệ sinh thái giải pháp bao gồm: văn hóa môi trường, đầu tư cho khoa học (Q. H. Vuong, 2018), thông tin truyền thông, hạ tầng công nghệ, và sự thực thi kỷ luật (Khuc, 2022). 


\section{Tài liệu tham khảo:}

Barnes, D. K. A., Galgani, F., Thompson, R. C., \& Barlaz, M. (2009). Accumulation and fragmentation of plastic debris in global environments. Philosophical Transactions of the Royal Society B: Biological Sciences.

Brooks, A. L., Wang, S., \& Jambeck, J. R. (2018). The Chinese import ban and its impact on global plastic waste trade. Science Advances, 4(6), 1-8. https://doi.org/10.1126/sciadv.aat0131

Hanh, H. T. H. (2022). Nguyên nhân và giải pháp cho vấn đề ô nhiễm và cạn kiệt nguồn nước. OSF Preprints. https://doi.org/10.31219/osf.io/ph2rs

Jambeck, J. R., Geyer, R., Wilcox, C., Siegler, T. R., Perryman, M., Andrady, A., Narayan, R., \& Law, K. L. (2015). Entradas de residuos plásticos desde la tierra al océano. Ciencia, 347(6223), 768-771.

http://www.sciencemag.org/cgi/doi/10.1126/science.1260879\%0Ahttps://www.s ciencemag.org/lookup/doi/10.1126/science.1260352

Khuc, Q. Van. (2021). Environmental culture thoughts to make a better world for our nature and children. OSF Preprints. https://doi.org/10.31219/osf.io/g5zex

Khuc, Q. Van. (2022). Về khả năng ứng dụng của hệ xử lý thông tin 3D và nguyên lý bán dẫn giá trị trong tìm kiếm giải pháp cho vấn đề ô nhiễm môi trường và biến đổi khí hậu ở Việt Nam. Tạp Chí Kinh Tế và Dự Báo, 1-5.

https://kinhtevadubao.vn/ve-kha-nang-ung-dung-cua-he-xu-ly-thong-tin-3d-vanguyen-ly-ban-dan-gia-tri-trong-tim-kiem-giai-phap-cho-van-de-o-nhiem-moitruong-va-bien-doi-khi-hau-o-viet-nam-20840.html

Khuc, Q. Van, Tran, B. Q., Meyfroidt, P., \& Paschke, M. W. (2018). Drivers of deforestation and forest degradation in Vietnam: An exploratory analysis at the national level. Forest Policy and Economics, 90(February), 128-141. https://doi.org/10.1016/j.forpol.2018.02.004

La, V. P. et al. (2020). Policy response, social media and science journalism for the sustainability of the public health system amid the COVID-19 outbreak: The vietnam lessons. Sustainability (Switzerland), 12(7). https://doi.org/10.3390/su12072931

Mont-Saint-Hilaire, Q. (2019). Government of Canada taking action to reduce plastic pollution. Prime Minister of Canada.

Nguyen, M.-H., \& Vuong, Q.-H. (2021). Evaluation of the Aichi Biodiversity Targets: the international collaboration trilemma in interdisciplinary research. Pacific Conservation Biology. https://doi.org/10.1071/pc21026

Nguyễn Việt Cường. (2020). Nỗ lực của Nhật Bản trong xử lý rác thải nhựa. Tạp Chí Môi Trường, Tạp trí Môi trường.

Peng, Y., Wu, P., Schartup, A. T., \& Zhang, Y. (2021). Plastic waste release caused by COVID-19 and its fate in the global ocean. Proceedings of the National Academy of Sciences of the United States of America, 118(47). https://doi.org/10.1073/pnas.2111530118

Rattner, N. (2020). As coronavirus restrictions drag on, Americans shift online spending from stockpiling to entertainment. $C N B C$.

Tập, T. biên. (2020a). Rác thải nhựa trên biển - Nỗi ám ảnh của đại dương và sinh vật biển. An Phat Holdings.

Tập, T. biên. (2020b). Tác hại khôn lường của rác thải nhựa đối với môi trường và cuộc sống. An Phat Holdings. 
Thu Thủy. (2020). Những nỗ lực của Việt Nam trong phòng chống ô nhiễm chất thải nhựa đại dương. In Tổng cục biển và hải đảo Việt Nam.

Van Khuc, Q., Phu, T. V., \& Luu, P. (2020). Dataset on the Hanoian suburbanites' perception and mitigation strategies towards air pollution. Data in Brief, 33, 106414. https://doi.org/10.1016/j.dib.2020.106414

Vuong, Q. H., et al. (2022). Covid-19 vaccines production and societal immunization under the serendipity-mindsponge-3D knowledge management theory and conceptual framework. Humanities and Social Sciences Communications, 9, forthcoming.

Vuong, Q.-H. (2021). Western monopoly of climate science is creating an eco-deficit culture. Economy, Land \& Climate Insight, 11, 1-9. https://elcinsight.org/western-monopoly-of-climat

Vuong, Q. (2020). From children's literature to sustainability science, and young scientists for a more sustainable Earth From children's literature to sustainability science, and young scientists for a more sustainable Earth. Journal of Sustainability Education, 24(December), 2019-2021.

Vuong, Q. H. (2018). The (ir)rational consideration of the cost of science in transition economies. Nature Human Behaviour, 2(1), 5. https://doi.org/10.1038/s41562-017-0281-4

Vuong, Q. H. (2021). The semiconducting principle of monetary and environmental values exchange. Economics and Business Letters, 10(3), 284-290. https://doi.org/10.17811/ebl.10.3.2021.284-290

Vuong, Q. H., Ho, M. T., Nguyen, M. H., Pham, T. H., Vuong, T. T., Khuc, Q., Ho, H. A., \& La, V. P. (2021). On the environment-destructive probabilistic trends: A perceptual and behavioral study on video game players. Technology in Society, 65(February), 101530. https://doi.org/10.1016/j.techsoc.2021.101530

Vuong, Q., Phu, T. V., Le, T. T., \& Khuc, Q. Van. (2021). Exploring Inner-City Residents' and Foreigners' Commitment. Data, 6(39). https://doi.org/10.17632/8sv4j2r9wk.2

WHO. (2020). Shortage of personal protective equipment endangering health workers worldwide. World Healthy Organization. https://www.who.int/news/item/03-03-2020-shortage-of-personal-protectiveequipment-endangering-health-workers-worldwide

Yudell, M., Roberts, D., DeSalle, R., \& Tishkoff, S. (2020). NIH must confront the use of race in science. Science, 369(6509), 1314-1315. https://doi.org/10.1126/SCIENCE.ABD9925 